\title{
THE DYNAMICS OF BENZENE MOLECULES IN LIQUID CARBON TETRACHLORIDE SOLUTION*
}

\author{
D. Lewandowska, Z. Trumpakaj, A. Śliwiński, J. Grzywacz \\ Institute of Experimental Physics, University of Gdańsk \\ Wita Stwosza 57, 80-952 Gdańsk, Poland \\ (Received April 23, 1990; in final version May 8, 1991)
}

\begin{abstract}
Results of the investigations of $\mathrm{C}_{6} \mathrm{H}_{6}$ and $\mathrm{CCl}_{4}$ binary solutions by two methods: dispersion of light and viscometry, as functions of the concentration and temperature are reported.
\end{abstract}

PACS numbers: 51.20.+d, 33.20.Fb, 35.20.Yh

\section{Introduction}

It is well-known that the dynamics of molecules depends both on temperature and the environment. When a molecule is in liquid solution, the above mentioned factors can influence strongly the changes of the intermolecular forces. Molecules which have neither charge nor constant dipole moment interact mainly by Lennard-Jones forces which strongly depend on the shape of molecules and their interdistances. Benzene solution in carbon tetrachloride is a typical model of such a system.

The aim of our measurements was to obtain information on the influence of $\mathrm{CCl}_{4}$ spherical molecules on the dynamics and orientation of plane $\mathrm{C}_{6} \mathrm{H}_{6}$ molecules.

\section{The methods of investigation and preparation of samples}

In the present paper the investigations of the $\mathrm{C}_{6} \mathrm{H}_{6}$ and $\mathrm{CCl}_{4}$ binary solutions by two methods: dispersion of light and viscometry as functions of concentration and temperature, are reported. 
The investigation methods applied were described in previous papers $[1,2,3]$. They enable Rayleigh's time of rotational relaxation to be obtained with an accuracy of $4 \%$, and the coefficient of viscosity with the accuracy of $1 \%$. For the concentration measurements, solvents of spectral purity (Fluka) have been additionally purified by chemical methods [4]. The measurements were carried out as functions of concentration (for $\tau, \eta$ ) and temperature (for $\eta$ ), and stabilized with an accuracy up to $0.3 \mathrm{~K}$.

The concentration changes in $I(\omega)$ shape and in the intensities of depolarized Rayleigh's spectrum, as well as the concentration and temperature changes of viscosity were determined.

\section{Results of the measurements}

\subsection{Dispersion of light}

The intensity in the maximum $I_{0}$ of the depolarized Rayleigh's spectrum $I(\omega)$, where

$$
I(\omega)=I_{0} /\left(1+\tau_{\text {RAY }}^{2} \omega^{2}\right)
$$

depending on benzene concentration $x$ in $\left(\mathrm{C}_{6} \mathrm{H}_{6}+\mathrm{CCl}_{4}\right)$ solution at $292 \mathrm{~K}$ is shown in Fig. 1.

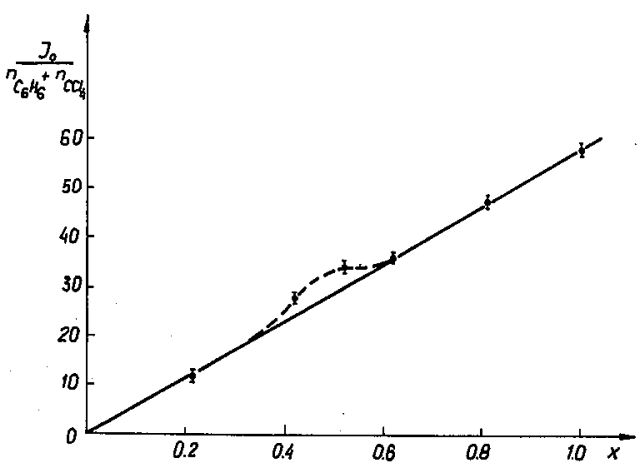

Fig. 1. The dependence of intensity of Rayleigh's band in the maximum (when this band is approximated by the Lorentz's shape) as a function of benzene concentration.

Figure 2 shows an example of the relative intensity of $I(\omega)$ spectrum, thus enabling a conclusion to be drawn that the main contribution to the intensity of dispersed light is due to benzene molecules. At concentration 0.5 , a deviation from the linear dependence of $I_{0}(x)$ is observed.

The relaxation time of benzene molecules in $\mathrm{CCl}_{4}$ solution was determined from the half width of the spectrum. The results are shown as a function of concentration in Fig. 3. The decrease in the relaxation time with increasing $\mathrm{C}_{6} \mathrm{H}_{6}$ 


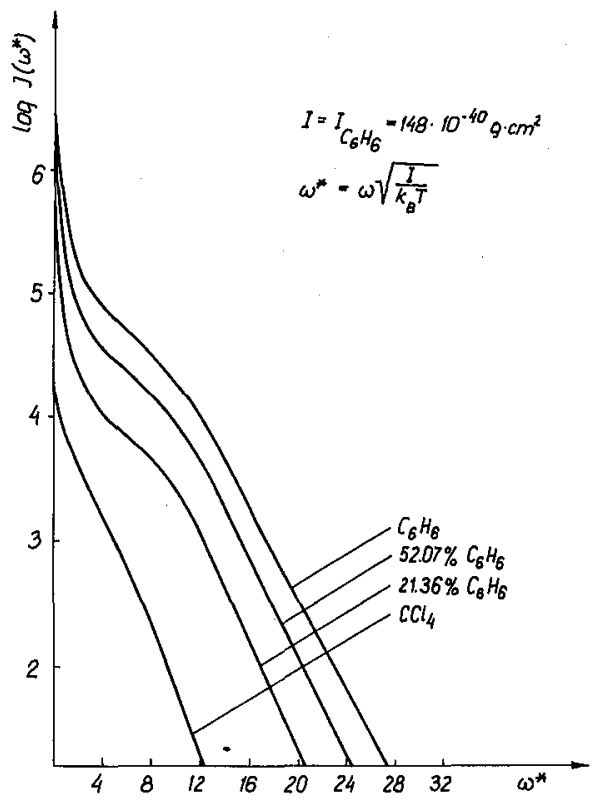

Fig. 2. The relative spectral intensity of Rayleigh's spectrum of benzene solutions in $\mathrm{CCl}_{4}$.

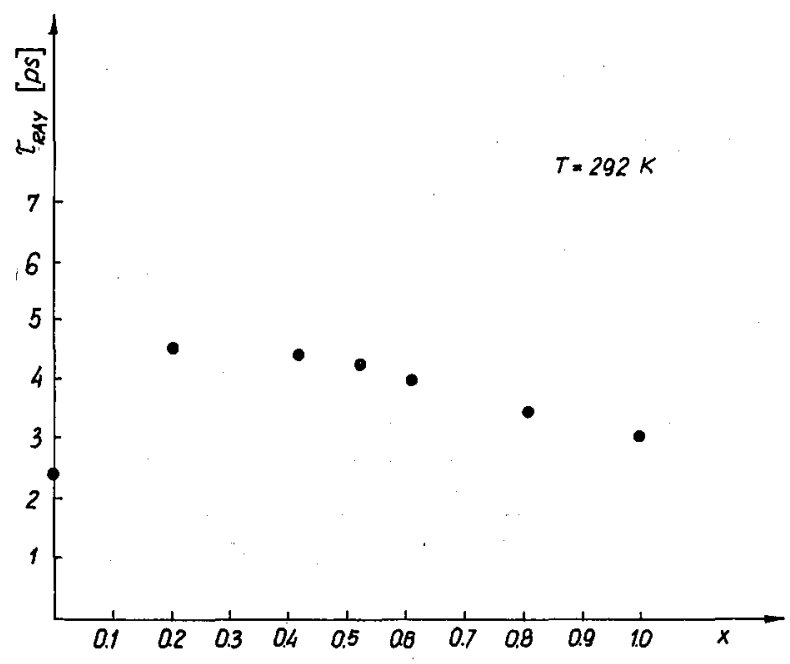

Fig. 3. The dependence of correlation time of benzene molecules as a function of $\mathrm{C}_{6} \mathrm{H}_{6}$ concentration in $\mathrm{CCl}_{4}$. 
concentration in the solution is observed. Similar concentration changes of $\tau_{\text {RAY }}$ in $\mathrm{CS}_{2}+\mathrm{CCl}_{4}$ and $\mathrm{CH}_{3} \mathrm{CN}+\mathrm{CCl}_{4}$ solutions were observed by Versmold [2].

\subsection{Shear viscosity coefficient}

An example of the temperature changes in the shear viscosity coefficient of $\mathrm{C}_{6} \mathrm{H}_{6}+\mathrm{CCl}_{4}$ solution is shown in Fig. 4. All solutions display temperature changes

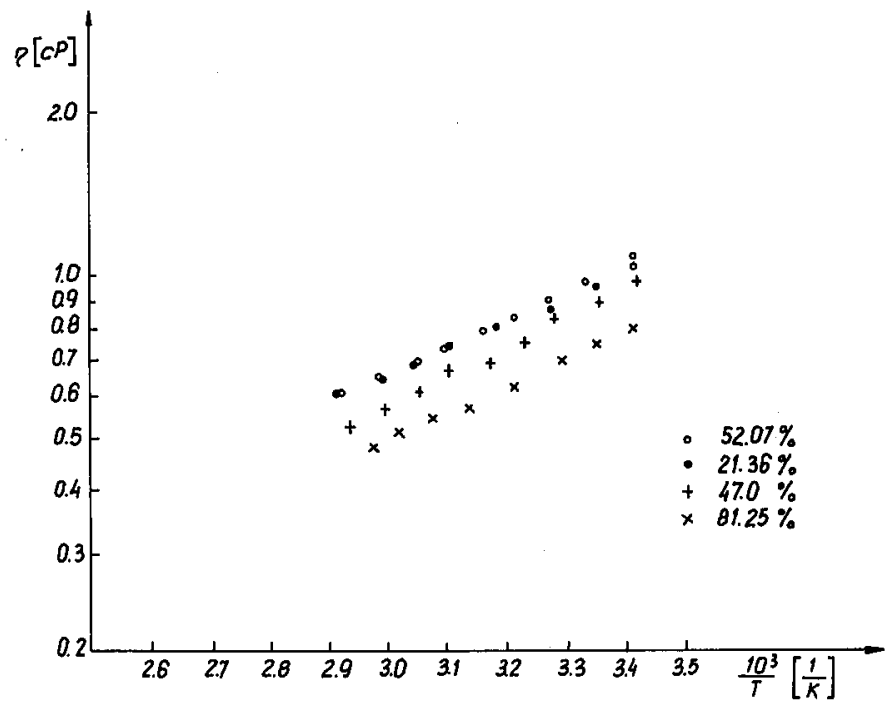

Fig. 4. The temperature changes of viscosity coefficient of benzene solutions in $\mathrm{CCl}_{4}$.

according to the Arrhenius equation:

$$
\eta(t)=\eta_{0} \exp \left(E_{\mathrm{a}} / R T\right) .
$$

Coefficients $E_{\mathrm{a}}$ and $\eta_{0}$ calculated on the basis of experimental data for several concentrations are listed in Table I.

\section{TABLE I}

The activation energy, $E_{\mathrm{a}}$ and viscosity, $\eta_{0}$, as functions of concentration of benzene, $x$, in $\mathrm{CCl}_{4}$.

\begin{tabular}{c|r|c}
\hline \hline$x\left(\mathrm{C}_{6} \mathrm{H}_{6}\right) \%$ & $E_{\mathrm{a}}(\mathrm{kJ} / \mathrm{mol})$ & $\eta_{\mathrm{o}}\left(10^{-4} \mathrm{P}\right)$ \\
\hline 21.4 & $9.08 \pm 0.2$ & 2.4 \\
47.0 & $10.36 \pm 0.3$ & 1.3 \\
52.1 & $9.66 \pm 0.1$ & 2.0 \\
81.3 & $9.07 \pm 0.2$ & 1.5
\end{tabular}

The changes in the activation energy as a function of concentration can be observed. 
According to Westmeier [5] and Fialkow [6], additional information on the processes occurring in the system can be obtained by analysing the temperature coefficient of viscosity:

$$
\alpha=\frac{\mathrm{d} \eta}{\mathrm{d} T} \quad \text { and } \quad \beta=\frac{\alpha}{\eta} .
$$

Figure 5 shows the temperature dependence of $\alpha$. A decrease of $\alpha$ with the increase

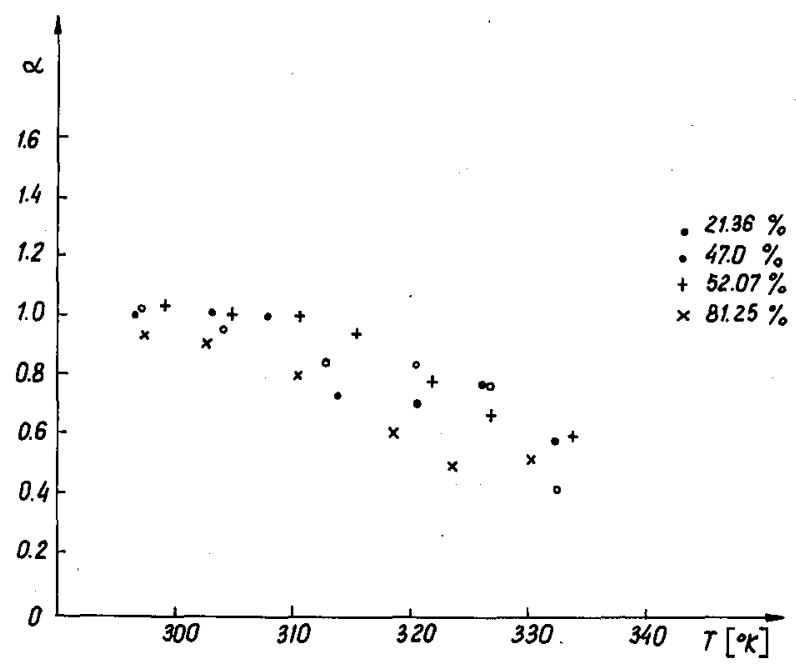

Fig. 5. The temperature changes of $\alpha$ coefficient for the benzene solutions in $\mathrm{CCl}_{4}$.

of temperature is observed. The isotherms of the concentration dependent $\alpha$ coefficient are shown in Fig. 6 . The maximum of $\alpha$ coefficient occurs at a concentration of about $50 \%$.

For all solutions investigated $\beta$ decreases with the increase of temperature. A total temperature change of $\beta$ for a given concentration does not exceed $30 \%$.

\section{Discussion of results}

The comparison of $I_{0}(x)$ and $\alpha(x)$ shown in Figs. 1 and 6 enables the correlation between the above values to be found. Other authors $[2,5]$ suggest that the existence of maximum in the $\alpha(x)$ curves proves that an intermolecular complex is created. Generally, the relation between the intensity of dispersed light and the degree of molecular orientation in the medium is known. The decrease of the disorientation results in the increase in the intensity of dispersed light. It can be presumed that around the concentration $x=0.5$ a structural change stronger than that for other concentrations in short-range ordering occurs.

The mean intermolecular distances calculated by the approximation with "a face centered cubic structure" amount to $6.097 \AA$ and $5.931 \AA$ for pure $C^{\circ} l_{4}$ and $\mathrm{C}_{6} \mathrm{H}_{6}$, respectively. 


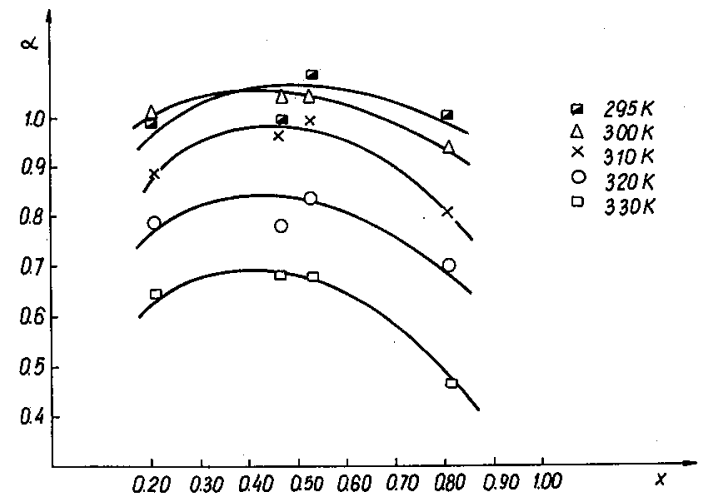

Fig. 6. The isotherm of $\alpha$ coefficient in the dependence on concentration.

The geometrical dimensions of the benzene molecule are:

$$
\begin{aligned}
& \text { diameter }_{\max }=6.92 \AA, \\
& \text { diameter }_{\min }=3.40 \AA .
\end{aligned}
$$

Mutual relations of the above values suggest that $\mathrm{C}_{6} \mathrm{H}_{6}$ molecules in the environment of $\mathrm{CCl}_{4}$ molecules cannot rotate freely around the axis perpendicular to the maximum diameter. The $\mathrm{CCl}_{4}$ molecules can stimulate the relative order of the $\mathrm{C}_{6} \mathrm{H}_{6}$ molecules.

According to paper [7], there exist four basic association types of planar symmetric top molecules.

In the case of solutions investigated, the parallel orientation of $\mathrm{C}_{6} \mathrm{H}_{6}$ molecules is the most probable, which is also in agreement with dependence of the relaxation time on concentration shown in Fig. 3. This dependence, which is in accordance with the measurements of Alms et al. [8] evidences the increase of benzene self-association with the decrease of its concentration.

\section{Conclusion}

The increase of the correlation time with the decrease of concentration of the $\mathrm{C}_{6} \mathrm{H}_{6}$ molecules in $\mathrm{CCl}_{4}$ suggests that rotational dynamics of $\mathrm{C}_{6} \mathrm{H}_{6}$ molecules decreases when the number of $\mathrm{CCl}_{4}$ molecules in the medium increases. The changes of the translational dynamics of molecules in solution have been observed which, in particular, agrees with the results listed in Table I and Fig. 6.

\section{References}

[1] D. Lewandowska, C. Lewa, Acta Phys. Pol. A59, 295 (1981).

[2] H. Versmold, Ber. Bunsenges. Phys. Chem. 82, 451 (1978). 
[3] Z. Trumpakaj, D. Lewandowska, Acta Phys. Pol. A70, 5 (1986).

[4] I. Vogel, Preparatyka Organiczna, Wyd. Nauk.-Techn. Warszawa 1984 (in polish).

[5] S. Westmeier, Z. Phys. Chem. 257, 5 (1976).

[6] J. Fiałkow, Chemia Fiz. Roztworów Niewodnych, PWN, Warszawa 1983 (in polish.)

[7] J.N. Murrell, V.M.S. Gill, Trans. Faraday Soc. 61, 402 (1965).

[8] G.R. Alms, D.R. Bauer, J.I. Brauman, R. Pecora, J. Chem. Phys. 59, 5310 (1973).

[9] D.A. Long, F.S. Murfin, E.L.Thomas, Trans. Faraday Soc. 59, 12 (1963). 